\title{
A 3-Axis Force Balanced Accelerometer Using a Single Proof-Mass
}

\author{
Mark A. Lemkin, Bernhard E. Boser, David Auslander ${ }^{*}$ Jim Smith $^{* *}$ \\ BSAC, 497 Cory Hall, U.C. Berkeley, Berkeley CA 94720 \\ ${ }^{*}$ Dept. of Mechanical Engineering, U.C. Berkeley, Berkeley CA 94720 \\ ** Sandia National Laboratories P.O. Box 5800, Albuquerque, NM 87185-1080

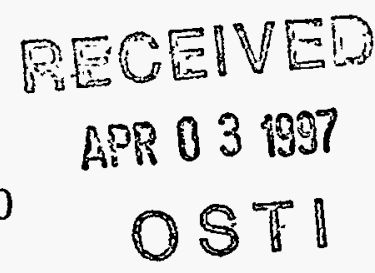

\section{SUMMARY}

This paper presents a new method for wideband force balancing a proof-mass in multiple axes simultaneously. Capacitive position sense and force feedback are accomplished using the same air-gap capacitors through time multiplexing. Proof of concept is experimentally demonstrated with a single-mass monolithic surface micromachined 3-axis accelerometer.

\section{INTRODUCTION}

Recent work has shown the feasibility of sensing acceleration in three axes with a single proof-mass, in both open loop [1,2] and closed loop configurations [3]. Closed Ioop operation has the ability to extend dynamic range, increase linearity, flatten frequency response, and improve cross-axis rejection [3]. Reference [3] measures position and force balances the proof-mass in a method similar to [4], using multiple carrier frequencies for different axes. While the results from [3] are impressive, this topology provides limited loop bandwidth, is limited to a singleended electrical interface, and is sensitive to nonlinearities in the analog signal processing circuitry which can cause intermodulation of the different carrier frequencies.

Use of a sigma-delta feedback loop for force balancing of micromachined accelerometers has proven to be a highly effective topology as shown in $[5,6,7]$. The system presented in this paper uses three $\Sigma \Delta$ feedback loops with a differential sense interface, of which a single-axis version is described in [7], to realize a high bandwidth, fully differential 3-axis accelerometer in a digital CMOS technology. Using a standard digital CMOS technology allows integration of the sensor with digital signal processing on a single silicon die. Extension of this method to six degree of freedom (DOF) servo control of translational and rotational modes is easily accomplished, even in a single structural poly layer, through time multiplexing of capacitor configuration.

Section II describes the 3-axis mechanical sense-element and how multi-axis sensing is realized. In Section III system level implementation of the sigma-delta loop for a single-axis is presented, including a brief discussion of electrical implementation. Section IV discusses issues particular to implementation of 3-axis servoing. Experimental measurements from a fabricated device are presented in section $\mathrm{V}$.

\section{3-AXIS PROOF-MASS}

In a mechanical spring-mass system deflection of the proof-mass in response to an input acceleration is proportional to the inverse of the resonant frequency squared, for frequencies below resonance. Thus, in order to measure translational acceleration in three axes it is necessary to have a mechanical structure compliant along all three axes.

Figure 1 shows the $2.3 \mu \mathrm{m}$-thick mechanical sense-element used for 3-axis sensing, with FEM simulations of the lateral ( $\mathrm{x}$ - and $\mathrm{y}$-axes) and out of plane (z-axis) resonant modes. Resonant frequencies are chosen to be approximately equal in all three axes for comparable performance in all 3 axes. Quad symmetry of the proofmass about the z-axis, minimizes sensitivity to off-axis accelerations. When a lateral acceleration is applied to the substrate, comb finger gaps change and cause an imbalance in the capacitive half bridge shown in Figure 2a [5]. By laying out comb fingers in a common centroid offaxis accelerations become a common mode signal, achieving first order rejection of both translational and rotational off-axis accelerations [5].

Under an applied z-axis acceleration the proof-mass moves out of plane, causing a change in the parallel plate capacitance formed between the center of the proof-mass and a bottom plate made from ground plane polysilicon, as shown in Figure $2 b$. To achieve a differential $z$-axis sense interface a reference capacitor, made from a separate mechanical structure for good matching over temperature, is used in the half bridge.

In capacitive sensing topologies one side of the capacitive bridge is typically driven with a low impedance voltage source, while the other side of the bridge is connected to

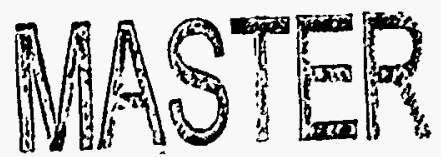




\section{DISCLAIMER}

This report was prepared as an account of work sponsored by an agency of the United States Government. Neither the United States Government nor any agency thereof, nor any of their employees, make any warranty, express or implied, or assumes any legal liability or responsibility for the accuracy, completeness, or usefulness of any information, apparatus, product, or process disclosed, or represents that its use would not infringe privately owned rights. Reference herein to any specific commercial product, process, or service by trade name, trademark, manufacturer, or otherwise does not necessarily constitute or imply its endorsement, recommendation, or favoring by the United States Government or any agency thereof. The views and opinions of authors expressed herein do not necessarily state or reflect those of the United States Government or any agency thereof. 


\section{DISCLAMMIRR}

Portions of this document may be illegible in electronic image products. Images are produced from the best available original document. 


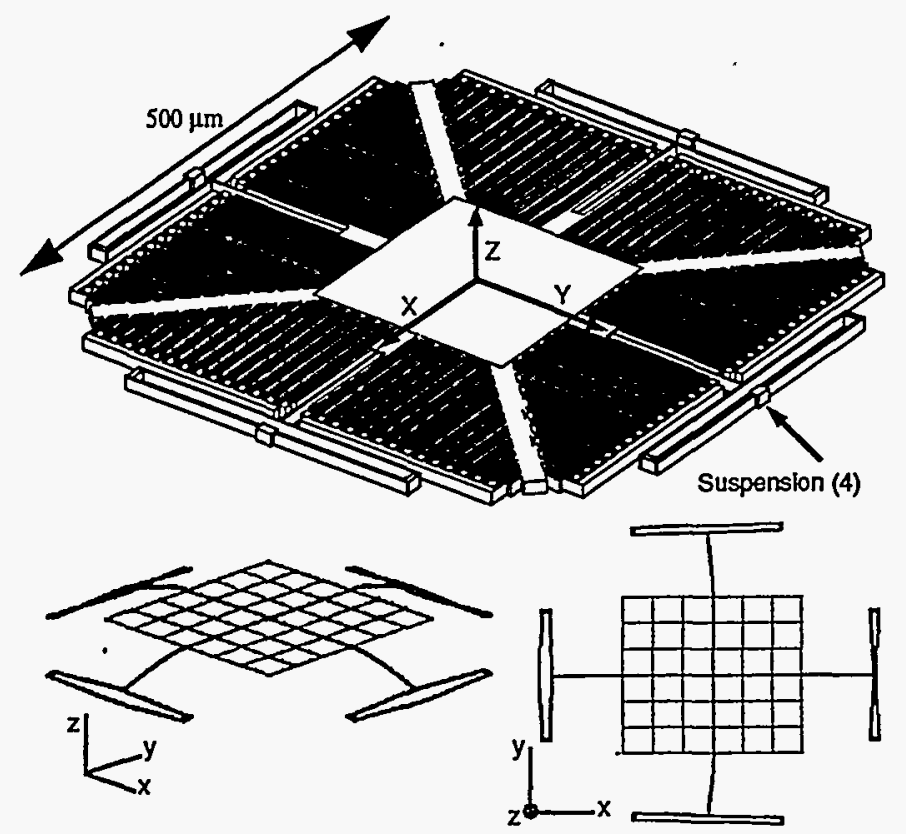

Figure 1: Undeformed $2.3 \mu \mathrm{m}$ thick structure (top). PATRAN simulations of deformation due to applied $Z$-axis (left bottom) and $\mathrm{X}$-axis (right bottom) accelerations are shown.

interface circuitry. A schematic diagram of the complete mechanical sensor is shown in Figure $2 c$. In this figure the proof-mass is a common node between all of the capacitive half bridges. By driving the proof-mass with a low impedance voltage source, as discussed in Section IV, position sense circuitry for all three axes may be decoupled thereby avoiding potential cross talk between different axes.

\section{III. $\Sigma \triangle$ FEEDBACK LOOP AND ELECTRICAL INTERFACE}

A sigma-delta feedback loop is used to provide force balancing and $A / D$ conversion of the analog input acceleration [6]. In this application the proof-mass acts as a second order integrator, integrating acceleration twice to position, providing the second order noise shaping necessary for a sigma-delta converter. A block diagram of the feedback loop for a single axis of this accelerometer is shown in Figure 3 [7]. Since this feedback loop is realized as a sampled data system, time multiplexing of capacitor function during position sensing and force feedback operations is possible. By utilizing all available comb fingers for both position sensing, resulting in a lowered noise floor, and force feedback, resulting in a larger full scale range, this accelerometer achieves a large dynamic range. The feedback loop operates in three phases, position sense, compare, and feedback.

During the position sense phase, position is sensed by applying a voltage pulse to the center node of the

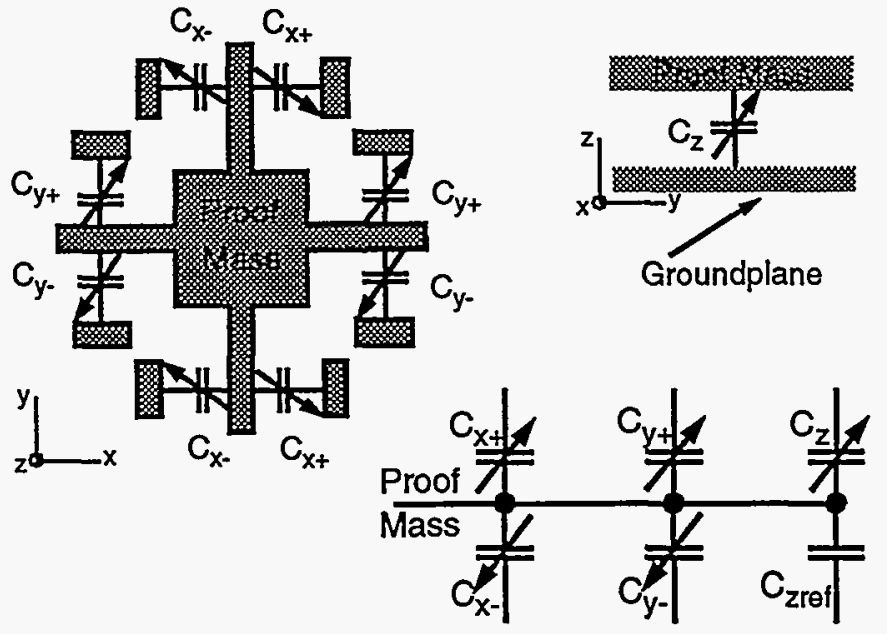

Figure 2: Proof-mass schematic for lateral acceleration measurement. $2 a$ (top left) schematic of $x$ - and $y$-axis capacitive half bridges, $2 b$ (top right) $z$-axis sense capacitor, $2 c$ (bottom) complete sensor schematic. Proof-mass is a common node for all capacitors.

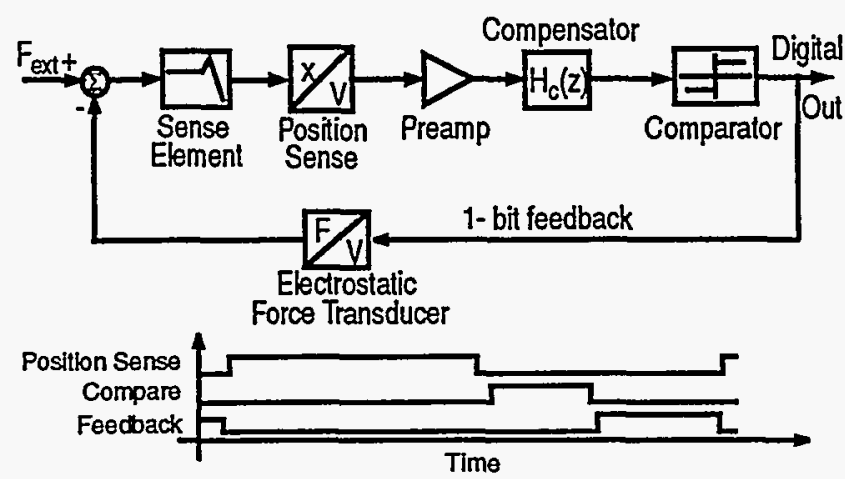

Figure 3: Single axis $\Sigma \Delta$ feedback loop and system level timing diagram.

capacitive half bridge and measuring the resulting imbalance with a charge integrator as shown in Figure 4. Note that as drawn this topology has two problems. Since the input common mode voltage of the opamp undergoes large voltage swings a large output offset, dependent on parasitic capacitance mismatch at the opamp input, will be present. Furthermore, offset and low frequency flicker noise from the electrical interface, represented by the voltage source $\mathrm{V}_{\text {error }}$ at the input, appear unattenuated at the output of the sensor. Input common mode swing may be greatly attenuated through the use of input common mode feedback [7], in which a voltage pulse of opposite sign and correct magnitude is applied through two feedback capacitors to both sides of the integrator input. Cancellation of opamp offset and flicker noise is achieved via correlated double sampling [7] in which errors are sampled and subtracted from the output.

During the compare phase the proof-mass is maintained at a constant voltage and the output of the position sense circuitry is sampled by the compensator. One-bit quantization of the compensator output is realized with a 


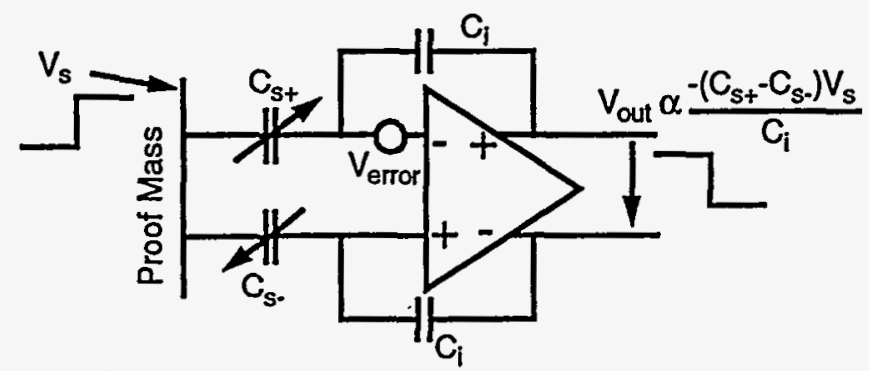

Figure 4: Charge integrator for a single axis. A voltage pulse applied to the center node of the capacitive half bridge results in a differential output voltage proportional to the difference in sense capacitance.
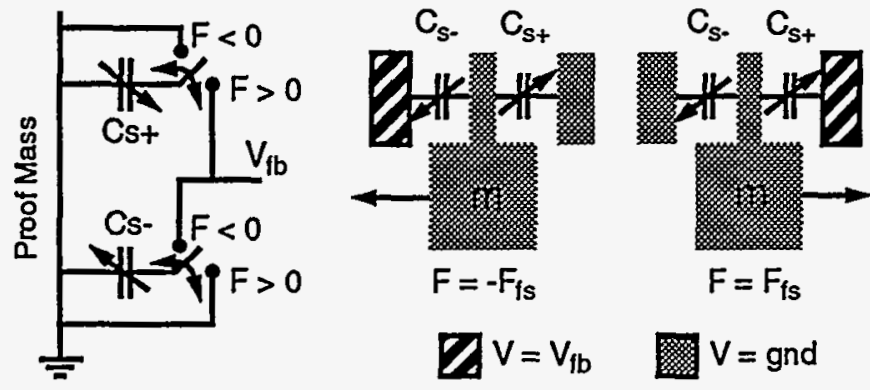

Figure 5: One-bit electrostatic feedback. Net feedback force is toward the finger held at $V_{f b}$.

fully differential regenerative latch [7].

Comparator output from the compare phase is used to determine correct direction of force feedback to the proofmass during the feedback phase. One-bit feedback is applied by grounding the proof-mass and applying a voltage $V_{\mathrm{fb}}$ across one side of the capacitive half bridge, while grounding the other end of the capacitive half bridge as shown in Figure 5. The net electrostatic feedback force is of constant magnitude over the entire feedback period and directed towards the capacitor plate held at $\mathrm{V}_{\mathrm{fb}}$.

\section{MULTI-AXIS SERVOING}

The mechanical sense element is designed such that the position sense circuitry for the $\mathrm{x}-, \mathrm{y}$-, and $\mathrm{z}$-axis capacitors is insensitive to off-axis deflections. Additionally, mechanical symmetry and capacitor layout of the sense element allows good selectivity between different axes when force feedback is applied. Because of the small electromechanical coupling between these orthogonal axes, feedback loop design may be undertaken one axis at a time, independent of other axes.

As discussed in Section III, the center node voltage of the single-axis capacitive half bridge is driven by a low impedance voltage source during all phases of operation. Looking at Figure $2 \mathrm{c}$ it is clear this center node is common to all three capacitive half bridges. By connecting a set of differential position sense and feedback circuitry to both

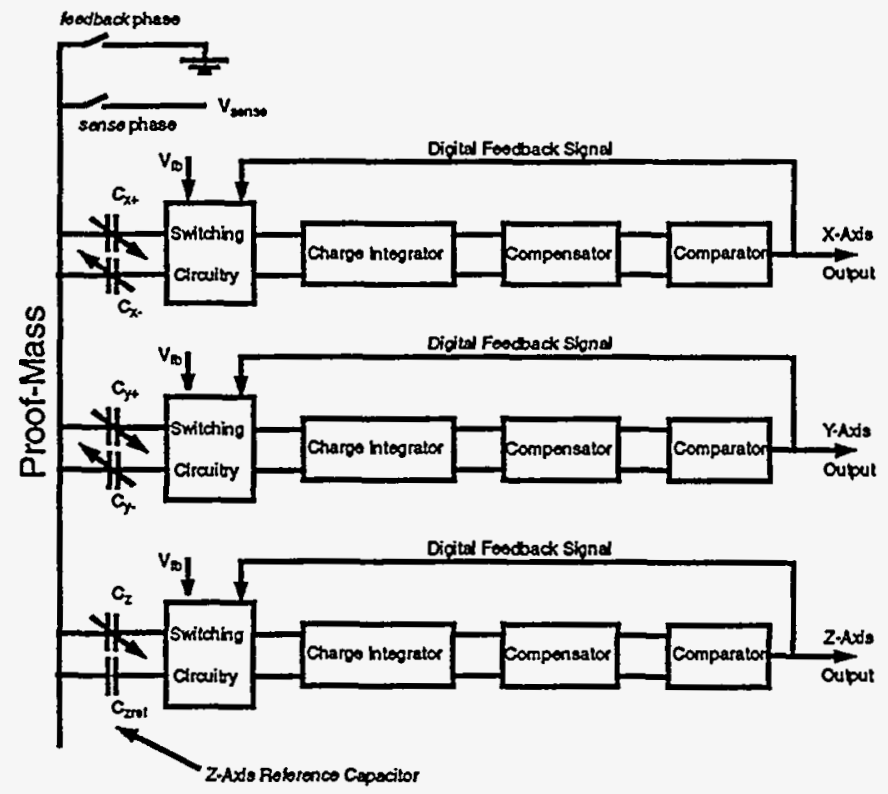

Figure 6: 3-Axis system diagram. Note the proof-mass is a common node for all capacitors, with the voltage at this node driven during both sense and feedback operations.

ends of each axis' half bridge, shown in Figure 6, multiaxis servoing can be accomplished. Synchronization of position sense and drive circuitry is necessary to account for clock skew originating from distribution of clocking phases to the three sets of independent circuitry. Synchronization is attained by waiting for acknowledgment pulses from each feedback loop to arrive at circuitry used for proof-mass switching before the proof-mass voltage is switched.

This same proof-mass may be converted to an $x-, y-, z$-axis angular accelerometer by creating slightly different proofmass /ground plane capacitors and rewiring the capacitors in the lateral axes as shown in Figure 7. The difference between the configuration shown in Figure 2 and Figure 7 is only in the wiring of the individual sense capacitor arrays. Thus the same mechanical sense element may be force balanced in $6 \mathrm{DOF}$ (translation and rotation in $x-, y-$, $z$-axes) by interleaving two periods in the sigma-delta feedback loop, during which the capacitors are rewired with MOSFET switches.

\section{RESULTS}

Figure 8 shows a micrograph of the $4 \mathrm{~mm} \times 4 \mathrm{~mm}$ fabricated sensor including the $2 \mu \mathrm{m}$ CMOS interface circuitry. The $0.2 \mu$-gram proof-mass is located in the center of the die. A reference capacitor is located beside the sense element, enabling use of differential $z$-axis circuitry. Table 1 summarizes important system parameters. Measured noise floor of all three axes is approximately $0.7 \mathrm{mG} / \mathrm{NHz}$. The noise floor is dominated 

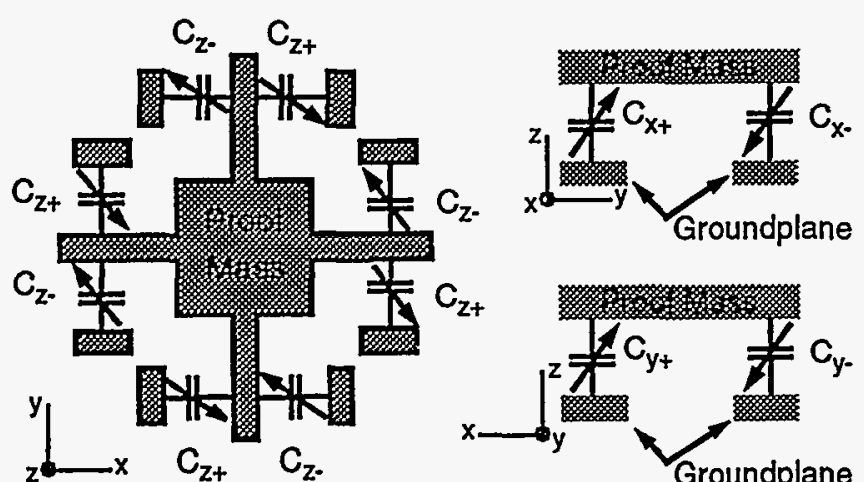

Figure 7: Capacitor configuration for rotational acceleration measurement of $z$ - (left), $x$ - (top right) and $y$-axis angular accelerations (bottom right).

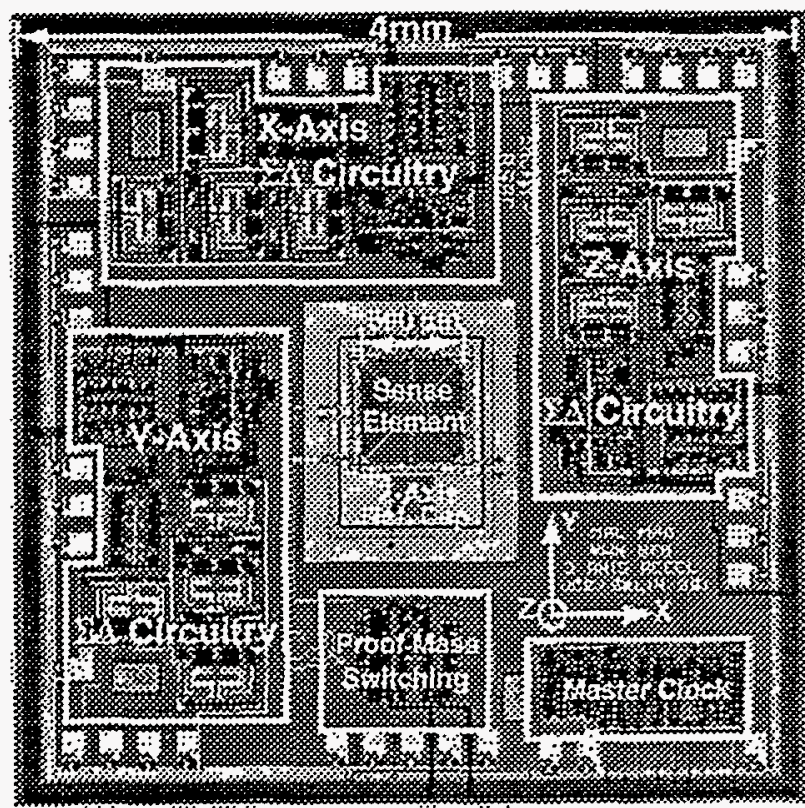

Figure 8: Die micrograph of fabricated 3-axis accelerometer.

by wiring resistance between the proof-mass and input to the charge integrator.

An obvious concem in any multi-axis accelerometer is the level of sensitivity to off-axis accelerations. Table 2 shows measured cross-axis sensitivity is very low for all axes, and well within tolerance of die to package misalignment. This low cross-axis sensitivity is possible because of the high orthogonality achievable with photolithography.

\section{DISCUSSION}

The ability to wideband force balance six-DOF simultaneously has applications in addition to multi-axis acceleration measurement. When operating in vacuum, to achieve a lower noise floor for instance, force balancing may be used to stabilize undamped modes. In this case feedback is used only to dampen certain structural modes and not directly for measurement of these modes, allowing smaller air-gap capacitors to be used. Other sensors requiring multi-axis sensing, such as vibratory rate
Table 1: System Parameters

\begin{tabular}{|c||l|l|l|}
\hline Parameter & \multicolumn{1}{|c|}{ X-Axis } & \multicolumn{1}{|c|}{ Y-Axis } & \multicolumn{1}{|c|}{ Z-Axis } \\
\hline \hline Natural Freq. & $6.6 \mathrm{kHz}$ & $6.6 \mathrm{kHz}$ & $5.2 \mathrm{kHz}$ \\
\hline Measured Full Scale & $\pm 11 \mathrm{G}$ & $\pm 11 \mathrm{G}$ & $\pm 5.5 \mathrm{G}$ \\
\hline Measured Noise & $0.73 \mathrm{mG} / \mathrm{Hz}$ & $0.73 \mathrm{mG} / \mathrm{Hz}$ & $0.76 \mathrm{mG} / \mathrm{Hz}$ \\
\hline Total Sense Cap. & $98 \mathrm{fF}$ & $98 \mathrm{fF}$ & $177 \mathrm{fF}$ \\
\hline Sensitivity & $0.24 \mathrm{fF} / \mathrm{G}$ & $0.24 \mathrm{fF} / \mathrm{G}$ & $0.82 \mathrm{fF} / \mathrm{G}$ \\
\hline
\end{tabular}

Table 2: Measured Cross Axis Sensitivity

\begin{tabular}{|c||c|c|c|}
\hline Input Axis & X-Output & Y-Output & Z-Output \\
\hline \hline$X$ & $\bullet$ & $-36 \mathrm{~dB}$ & $-39 \mathrm{~dB}$ \\
\hline $\mathrm{Y}$ & $-38 \mathrm{~dB}$ & & $-39 \mathrm{~dB}$ \\
\hline $\mathrm{Z}$ & $-47 \mathrm{~dB}$ & $-56 \mathrm{~dB}$ & \\
\hline
\end{tabular}

gyroscopes, may use this interface to both sense and drive the proof-mass around different axes. The digital output of the $\Sigma \Delta$ topology allows digital demodulation and quadrature correction of the gyro output signal.

$$
\text { Sandia Funding statement to be addact. }
$$
VII. REFERENCES

[1] Mineta, T. et. al., "Three-axis capacitive accelerometer with uniform axial sensitivities," Transducers '95 Dig. Tech. Papers, vol. 2, pp. 554-7.

[2] Andersson, G.I., "A novel 3-axis monolithic accelerometer", Transducers '95 Dig. Tech. Papers, vol. 2, pp. 558-61.

[3] Jono, K. et. al., "An electrostatic servo-type three-axis silicon accelerometer", Meas. Sci. Technol., Jan. 1995, vol.6, pp. 11-15.

[4] Analog Devices, "ADXL05 - $\pm 1 \mathrm{~g}$ to $\pm 5 \mathrm{~g}$ single chip accelerometer with signal conditioning," Datasheet, 1995, Norwood, MA 02062.

[5] Lemkin, M., Boser B.E., "A micromachined fully differential lateral accelerometer," CICC Dig. Tech. Papers, May 1996, pp. 315-318.

[6] Henrion, W., et. al., "Wide dynamic range direct digital accelerometer," IEEE Solid-State Sensor and Actuator Workshop, Hilton Head Island, SC, June 4-7, 1990 pp. 153157.

[7] Lemkin, M. et. al., "A 3-axis surface micromachined $\Sigma \Delta$ accelerometer," ISSCC Digest of Technical Papers, Feb. 1997, pp. 202-203.

Sandia is a multiprogram laboratory operated by Sandia Corporation, a Lockheed Martin Company, for the United States Department of Energy under contract DE-AC04-94AL85000. 